DOI: 10.17234/SRAZ.65.54

UDK: 81'246.2'27:314.7

Original scientific paper

Ricevuto il 2 maggio 2020

Approvato per la pubblicazione il 25 novembre 2020

\title{
Flussi migratori e identità: genere come parametro di oscillazioni identitarie
}

\author{
Karmen Tolić \\ Ivana Škevin Rajko \\ Università di Zara \\ ktolic@unizd.hr \\ iskevin@unizd.hr
}

\begin{abstract}
Il presente contributo analizza gli aspetti linguistici, sociali e identitari di uno dei fenomeni di spicco della società moderna: i flussi migratori. Attraverso 11 interviste semistrutturate si vuole fornire un panorama generale sulle dinamiche di (ri)costruzione identitaria di 6 donne e 5 uomini che sono emigrati dal loro paese d'origine (Croazia) per raggiungere l'Italia nei primi anni '90 e rimpatriare nel primo decennio del 2000. La nostra ricerca conferma che il genere è un parametro rilevante nei processi di costruzione identitaria e dimostra che le donne tendono ad usare le forme linguistiche standard e di prestigio e si identificano di più con l'italianità percepita come raffinata e colta, mentre gli uomini sono generalmente più legati alla propria nazionalità e alle proprie origini.
\end{abstract}

Parole chiave: migrazioni, identità, genere, Italia, Croazia.

\section{Introduzione e oggetto della ricerca}

Negli ultimi decenni i movimenti migratori hanno creato terreno fertile per la realizzazione di molte ricerche interdisciplinari, tra le quali spiccano quelle culturali, sociali e linguistiche. Essendo un punto di partenza per la costruzione di nuove identità, considerate dinamiche, "oscillanti" (De Fina 2007) o ibride (Bhabha 1990a, 1990b), le migrazioni in realtà permettono che l'identità (individuale o/e collettiva) diventi "un'attività processuale di identificazione che non trova soluzioni definitive, ma temporanee localizzazioni" (Petrosino 2004: 4; cfr. Bhabha 1990b).

Tra i demografi croati emerge l'idea per cui siano pochi i paesi al mondo ad aver subito una perdita della popolazione così rilevante come la Croazia (per es. Nejašmić 2014). Tre sono le ondate di emigrazione documentate in Croazia negli ultimi decenni (2014: 409-418): quella dal 1900 al 1948 dovuta alla crisi agricola in Europa; quella dal 1948 al 1991 durante la quale, oltre agli optanti che hanno rinunciato alla cittadinanza jugoslava a favore di quella italiana e che hanno 
fatto perdere alla Croazia 100.000 abitanti fino al 1961, l'emigrazione è avvenuta perlopiù per motivi economici; quella dal 1991 al 2001 avvenuta per motivi di guerra e a causa delle difficoltà economiche e sociali da essa provocate: si stima che la Croazia abbia perso in questo periodo 500.000 abitanti. Oggi come oggi, secondo i censimenti più recenti, si stima che in Italia ci siano circa 60.000 croati. $^{1}$

\section{Obiettivi e metodologia della ricerca}

L'obiettivo della presente ricerca è mettere a fuoco l'esperienza migratoria di undici persone emigrate da Zara in Italia nei primi anni Novanta e il loro ritorno in Croazia nel primo decennio del 2000, con lo scopo di verificare le dinamiche di (ri)costruzione identitaria che emergono dall'interazione verbale quale pratica in cui si manifesta l'identità linguistica e sociale (Edwards 2009; Coupland 2007). Il fenomeno che ci proponiamo di esplorare è l'influsso del genere sulla (ri)costruzione identitaria e l'emergere degli stereotipi associati al genere e all'appartenenza alla cultura e società italiana o croata.

A tal proposito ci siamo servite di un corpus di 11 interviste semistrutturate svolte con adulti croati (5 uomini e 6 donne), tutti originari di Zara. Il periodo di permanenza in Italia oscilla tra i 14 e i 23 anni e il periodo di permanenza in Croazia (dopo il rimpatrio) oscilla tra i 7 e i 12 anni al momento dell'intervista. L'emigrazione in Italia è avvenuta nella maggior parte dei casi per motivi economici ( 5 casi) e di guerra (4 casi), mentre due informanti (di seconda generazione) sono nati in Italia. Ogni informante sarà di seguito denominato per numero, con l'età approssimativa e con le lettere $\mathrm{M}$ per indicare il genere maschile o F per indicare il genere femminile (per esempio M01, F03 e così via). Ad ogni informante è stata data la possibilità di scegliere la lingua di svolgimento dell'intervista, fattore che può rilevare gli atteggiamenti linguistici che si nutrono nei confronti dei due codici (Dal Negro/Guerini 2007: 138): 8 informanti hanno optato per la lingua croata, mentre tre di loro hanno scelto la lingua italiana. Per motivi di spazio tutti gli esempi saranno presentati in lingua italiana, sebbene alcuni siano stati tradotti dal croato all'italiano.

\section{L'identità sospesa tra due paesi: genere come parametro di oscillazioni identitarie}

In quanto al genere come parametro di oscillazione identitaria, partiamo da una delle ipotesi sulla scelta delle varietà linguistiche più discusse e criticate (cfr. Cameron 1998) che riguarda la maggior tendenza delle donne verso le forme e le varietà di prestigio, standard e conservative rispetto agli uomini (Trudgill 1972; Labov 1991, 2001; Eckert/McConnell-Ginet 2003; Coupland 2007: 132; Berruto/Cerruti 2019) e una maggiore percezione delle proprie radici e del senso

1 https:/hrvatiizvanrh.gov.hr/hrvati-izvan-rh/hrvatsko-iseljenistvo/hrvatsko-iseljenistvou-talijanskoj-republici/760 (15/04/2020) 
di nazionalità negli uomini opposto al senso di libertà e autonomia economica che vogliono raggiungere le donne. Una delle ricerche che allo stesso tempo conferma e nega la prima ipotesi già menzionata è sicuramente quella di Labov (1991, 2000) che da un lato mostra che le donne preferiscono l'uso delle forme standard, e dall'altro le considera promotrici di innovazioni linguistiche (quindi dell'uso delle forme non standard). Quanto al desiderio di libertà e autonomia economica, evidente nel corpus raccolto, presupponiamo che le donne si aspettino di realizzarlo attraverso l'uso della varietà standard (cfr. Sankoff et al. 1989), che rappresenta la chiave d'accesso alla società e cultura italiane (cfr. Eckert/ McConnell-Ginet 2003) nonché una sorta di capitale simbolico (Bourdieu 1977a, $1977 b$ ) che assicura loro anche l'accesso al capitale economico. Dagli esempi risulta che in Italia le donne intervistate (si veda l'esempio 8) abbiano avuto più opportunità lavorative e un libero accesso al capitale economico, che generalmente (specialmente nel passato) era un diritto riservato agli uomini.

L'esempio 1 dell'informante F03 che vive in Croazia da 12 anni e ne ha trascorsi 17 in Italia, dimostra un atteggiamento propenso all'uso della varietà percepita come standard quale "modo di esprimersi che rappresenta un modello al quale avvicinarsi" (Dal Negro/Guerini 2007: 134), e mostra contrarietà verso l'uso della varietà dialettale.

(1) F03 (ca. 50 anni) [...] mi sforzavo a non parlare il romanesco, orribile per me.

In quanto alla percezione, le ricerche di Trudgill (1972) mostrano che gli uomini tendono a sovrastimare il carattere sub-standard delle proprie produzioni linguistiche associato al prestigio non esplicito (ovvero coperto), mentre quelle di Edwards (2009: 129-130) mostrano che le donne sovrastimano più spesso il carattere standard delle loro produzioni che spesso viene associato al concetto di prestigio aperto (Trudgill 1972). Per esempio, F04 che ha vissuto in Italia per 15 anni, e ancora oggi alterna periodi tra Italia e Croazia, afferma:

(2) F04 (ca. 35 anni) [usa il dialetto, N.d.A.] solo per necessità, ma cose brevi, piccole non per farci i discorsi.

Quando menziona il concetto di necessità intende la comunicazione con le persone anziane che si contraddistinguono per l'uso del dialetto e mostra la sua tendenza e preferenza per l'uso della varietà percepita come standard.

In virtù del prestigio coperto/aperto appena discusso possiamo affrontare anche la questione degli stereotipi e del loro influsso sulle dinamiche di (ri) costruzione identitaria. Da un lato i nostri informanti associano la croaticità alla rozzezza/grossolanità, alla chiusura mentale e comportamentale, alla mancata raffinatezza in termini di comportamento e in termini di cultura e in generale ad una mentalità chiusa e diversa da quella italiana (in senso negativo). Per esempio, F10 e F11 che vivono in Croazia entrambe da 9 anni e hanno vissuto in Italia la prima per 15 anni e la seconda per 20 anni affermano:

(3) F10 (ca. 40 anni) [In Italia, N.d.A.] Ho trovato molta educazione. In qualsiasi cosa. Le persone erano molto corrette. Mentre io qui, non so come descrivere, trovo i croati molto rozzi. 
(4) F11 (ca. 50 anni) Ma, sono brava gente. Chiacchierano di più. Magari sono più aperti, più espressivi. Come ti posso dire [...] Croati sono più rigidi, più chiusi. Almeno penso così.

Stereotipi simili si notano anche nelle risposte degli informanti che dichiarano di aver provato sempre nostalgia per la Croazia durante la loro permanenza in Italia. D'altro canto, l'italianità è associata alla raffinatezza e finezza, all'educazione, alla bellezza ed esteticità, all'apertura mentale e comportamentale e a una mentalità aperta e diversa da quella croata (in senso positivo). Per esempio, M02 che vive da 12 anni in Croazia ed ha vissuto in Italia per 14 anni afferma:

(5) M02 (ca. 50 anni) Mi ha sempre affascinato l'estetica negli italiani [...] loro hanno questa raffinatezza, hanno qualcosa di attraente.

Allo stesso modo F10 e F06 (che ha vissuto 23 anni in Italia e vive in Croazia da 9 anni) affermano:

(6) F10 (ca. 40 anni) ho notato anche questo in Italia [...] lì è tutto un po' più fine.

(7) F06 (ca. 50 anni) Ma perché lì sono, le persone sono più aperte, penso di essermi ritrovata in quella società, somigliavo più, più a loro.

Le ricerche di Eckert e McConnell-Ginnet (2003: 283-294) mostrano che le donne preferiscono essere associate alla raffinatezza, eleganza e finezza in termini linguistici e sociali. Questo è visibile anche nel nostro corpus, poiché le interlocutrici si dichiarano più legate alla cultura italiana rispetto agli interlocutori e si (auto)identificano come italiane, almeno in parte. F06 per esempio afferma:

(8) F06 (ca. 50 anni) Io penso di essere più italiana perché lì mi sono ritrovata in tutto e penso che quel mondo sia adatto a me [...] la mia vita era quella che vivevo lì, quindi la libertà $[. .$.$] vivi come vuoi vivere [. .$.$] io non volevo$ tornare per nulla al mondo [in Croazia, N.d.A.] stavo bene, avevo la mia libertà, la mia bella paga, potevo mantenermi da sola, avevo lo stipendio di un milione di lire e in più l'alloggio pagato.

Per quanto riguarda l'esperienza del rimpatrio (e quindi del ritorno in Croazia) F03, F06 e F10 manifestano la loro insoddisfazione:

(9) F03 (ca. 50 anni) Malissimo. Sono caduta in depressione [...] L'hai fatto per volontà, per amore ma proprio contro te stessa, contro me stessa assolutamente [...] Ma qualcosa dentro di me, lì ero completamente libera [...] ma va bene dai, qui la città è su misura, per la famiglia, non ne voglio neanche più parlare, ormai l'ho soppressa questa cosa.

(10) F06 (ca. 50 anni) Beh è stato terribile, i miei due anni, due anni di vita persi qui quando sono tornata.

(11) F10 (ca. 40 anni) Purtroppo sono tornata. Molto dispiaciuta. Perché una volta tornata qui, non avevo, non accettavo più questa mentalità croata. E quindi, il ritorno è stato molto duro. Bisogna, mi dispiace dirlo, abbassarsi con la mentalità, con la cultura con tutto per sopravvivere qui. 
Questi esempi dimostrano che le donne sono più legate dal punto di vista emotivo e culturale alla mentalità e alla cultura italiana, che a loro avviso offre più libertà, più possibilità lavorative, culturali e in generale uno stile di vita diverso e più affine alla loro personalità. L'ambiente italiano ha contribuito alla ricostruzione della loro identità sociale attraverso opportunità di lavoro più favorevoli, uno status economico più alto e uno stile di vita culturalmente più interessante e ricco. D'altro canto, gli uomini si dichiarano più legati alla loro origine croata, alle loro radici, come si vede negli esempi 12, 13 e 14. M01 ha vissuto in Italia per 15 anni e vive in Croazia da 12 anni, mentre M08 ha vissuto in Italia per 22 anni e vive in Croazia da 7 anni.

(12) M01 (ca. 35 anni) Sono croato assolutamente, è sempre stato così anche mentre vivevo in Italia [...] non siamo mai stati in famiglia, come dire, in dubbio sulla nostra nazionalità [...] a prescindere da tutta la situazione in Croazia [la guerra, N.d.A.].

(13) M02 (ca. 50 anni) Io nell'anima sono, per così dire di nazionalità, non sono nazionalista ma sono consapevole della mia nazionalità [...] Che io adesso faccia finta di essere italiano e in realtà non lo sono!?

(14) M08 (ca. 50 anni) [...] tu sei, tu vivi qui ma, ma la tua testa e il tuo cuore sono in Croazia.

Dalle trascrizioni analizzate, emerge una caratteristica comune che riguarda gli stereotipi più diffusi sugli uomini e sulle donne: le donne sono molto più accurate e dettagliate nelle risposte rispetto agli uomini, sono più concentrate al contenuto del racconto (Cheshire 2000, Eckert/McConnell-Ginet 2003) e in generale sono più sensibili e più cooperative nel relazionarsi con gli altri (Eckert 1990, Coates 1996, Eckert/McConnell-Ginet 2003), raccontando più facilmente e apertamente la loro storia ai ricercatori (Edwards 2009: 131).

\section{Conclusione}

Le dinamiche di (ri)costruzione identitaria sono legate a molteplici fattori, ma sono sempre sottoposte a "cambiamenti che riguardano nuove identità, il ritorno a quelle vecchie e la trasformazione di quelle esistenti" (Jenkins 2004: 8; Edwards 2009: 18). Tutti i nostri informanti hanno in comune il fatto di essere tornati in Croazia durante lo stesso periodo (tra il 2007 e il 2009) che coincide con la forte crisi economica che ha colpito l'Italia. Secondo la loro opinione sono due i motivi principali che li hanno spinti al rimpatrio: la crisi economica appena menzionata e la crescita dei figli, ovvero il desiderio di vederli stabiliti in Croazia dove "la vita è più semplice" (esempio 9).

I risultati confermano che le migrazioni permettono che l'identità subisca delle "temporanee localizzazioni" (cfr. Petrosino 2004; Bhabha 1990b), infatti i nostri informanti mostrano oscillazioni dall'identità croata di partenza, attraverso la ricerca (esempio 8) o il rifiuto (esempio 13) dell'identità italiana (linguistica e sociale) 
e attraverso le identità locali espresse nell'uso delle varietà dialettali dell'italiano o della varietà standard, per poi di nuovo trovarsi in cerca della loro identità croata (persa o soppressa) (esempi 10, 12 e 14) nella quale alcuni di loro si sono ritrovati subito, alcuni si cercano ancora, mentre altri riconoscono in sé una doppia identità.

Sono state confermate le ipotesi prestabilite riguardo al parametro del genere: le donne confermano di preferire l'uso delle forme standard (esempi 1 e 2), si riconoscono di più nell'identità italiana associata alla raffinatezza, criticando quella croata associata alla rigidità e chiusura mentale (esempi 7 e 8); gli uomini mostrano di essere molto legati alle loro origini e alla loro nazionalità (esempi 12,13 e 14). Le donne inoltre considerano la lingua e la cultura italiana una sorta di capitale simbolico che ha aperto loro le porte al capitale economico italiano, confermando che la raffinatezza che associano all'italianità sia strettamente connessa all'uso della varietà standard (Eckert/McConnel-Ginet 2013). Si può concludere quindi che l'uso della varietà standard (esempi 1 e 2) e l'italianità rappresentino indirettamente le categorie sociali a cui le donne vogliono appartenere e a cui vogliono essere associate (esempi da 3 a 7).

\section{Bibliografia}

Berruto, Gaetano / Cerruti Massimo (2019). Manuale di sociolinguistica. Con espansione online, Torino: Utet.

Bhabha, Homi K. (1990a). Introduction: narrating the nation, in: Nation and Narration [a cura di Homi K. Bhabha], London: Routledge, pp. 1-8.

Bhabha, Homi K. (1990b). DissemiNation: time, narrative and the margins of the modern nation, in: Nation and Narration [a cura di Homi K. Bhabha], London: Routledge, pp. 291-323.

Bourdieu, Pierre (1977a). The economics of linguistic exchanges, in: Social Science Information, 16, pp. 645-668.

Bourdieu, Pierre (1977b). Outline of a Theory of Practice, Cambridge: Cambridge University Press.

Cameron, Deborah, (1998). Gender, language and discourse: a review essay, in: Signs: Journal of Women in Culture and Society, 23, pp. 945-973.

Cheshire, Jenny (2000). The telling or the tale? Narratives and gender in adolescent friendship networks, in: Journal of Sociolinguistics, 4, pp. 236-262.

Coates, Jennifer (1996). Women Talk: Conversation Between Women Friends, Oxford: Blackwell.

Coupland, Nikolas (2007). Style: Language variation and identity, Cambridge: Cambridge University Press.

Dal Negro, Silvia / Guerini, Federica (2007). Contatto: Dinamiche ed esiti del plurilinguismo, Roma: Aracne.

De Fina, Anna (2007). Parlando di mangiare: l'identità come costruzione interazionale, in: La costruzione internazionale di identità. Repertori linguistici e pratiche discorsive degli italiani in Australia [a cura di Anna Ciliberti], Milano: Franco Angeli, pp. 68-89. 
Eckert, Penelope (1990). Cooperative competition in adolescent girl talk, in: Discourse Processes, 13, pp. 92-122.

Eckert, Penelope / McConnell-Ginet, Sally (2013). Language and Gender, Cambridge: Cambridge University Press.

Edwards, John (2009). Language and Identity: An introduction, Cambridge: Cambridge University Press.

Jenkins, Richard (2004). Social Identity, London: Routledge.

Labov, William (1991). The intersection of sex and social class in the course of linguistic change, in: Language Variation and Change, 2, pp. 205-251.

Labov, William (2001). Principles of Linguistic Change: Social Factors, Oxford: Blackwell.

Nejašmić, Ivo (2014). Iseljavanje iz Hrvatske od 1900. do 2001.: demografske posljedice stoljetnog procesa, in: Migracijske i etničke teme, 3, pp. 405-435.

Petrosino, Daniele (2004). Pluralismo culturale, identità, ibridismo, in: Rassegna italiana di sociologia. 45, 3, pp. 1-30.

Sankoff, David/ Cedergren, Henrietta J./ Kemp, William/ Thibault, Pierre/ Vincent, Diane (1989). Montreal French: language, class and ideology, in: Language Change and Variation [a cura di Ralph W. Fasold/ Deborah Schiffrin] Amsterdam: John Benjamins, pp. 107-118.

Trudgill, Peter (1972). Sex, covert prestige and linguistic change in the urban British English of Norwich, in: Language in society 1.2, pp. 179-195.

\section{Migrations and identity: gender as a parameter of identity variation}

This paper, based on a sociolinguistic fieldwork which comprised semi-structured interviews with 11 participants, analyses the linguistic, social and identity aspects of migratory movements. The objective of the paper is to provide general insight into the identity construction of 6 Croatian women and 5 Croatian men who emigrated to Italy in the early 1990s and repatriated to Croatia in the first decade of the 2000s. Our research confirms gender as a relevant parameter of identity construction and confirms that female interviewees, during their stay in Italy, preferred the use of forms perceived as standard language forms. It also shows that women generally perceive the Italian social and linguistic context as more refined then the Croatian one and as the one they want to be identified with. Men, on the contrary, report on a stronger attachment to their Croatian origins.

Key words: migrations, identity, gender, Italy, Croatia 
